\title{
On a Conjecture of P. Jorgensen and W. Klink
}

\author{
By
}

\section{Bernard HELFFER*}

In $[\mathrm{J} . \mathrm{K}]$, the authors conjecture that if $\lambda_{n}(\alpha)$ is the series of the eigenvalues of the operator:

$$
H_{\alpha}=-\Delta_{x}+\left(\alpha+x_{1} x_{2}\right)^{2} \quad \text { in } \mathbb{R}^{2}(\alpha \in \mathbb{R})
$$

then each of the $\lambda_{n}(\alpha)$ is continuous in $\alpha$.

It is relatively easy to prove this conjecture using the technics of $[\mathrm{HE}-\mathrm{NO}]_{3}$ combined with the more classical technics of Kato [KA]. Using technics of Helffer-Sjöstrand $[\mathrm{HE}-\mathrm{SJ}]_{1,2}$, we study also the asymptotic behavior of $\lambda_{n}(\alpha)$ for $|\alpha| \rightarrow \infty$. Let us first recall briefly some results which are used in the proof of the hypoellipticity of invariant homogeneous operators on stratified nilpotent groups [HE$\mathrm{NO}_{3}$. Let us consider a stratified nilpotent Lie algebra $\mathbb{S}=\mathbb{S}_{1} \oplus$... $\oplus \mathscr{S}_{r}$ of rank $r$ and $P=\sum_{2} Y_{2}^{2} \in \mathfrak{H}_{2}(\mathbb{S})$ where $Y_{i}$ is a basis of $\mathbb{S}_{1}$. For each irreducible $\pi \in \hat{G}, \pi(P)$ is an essentially self-adjoint operator starting from $C_{\pi}^{\infty}$ and the self-adjoint extension has as domain the Sobolev space $H_{\pi}^{2}=\left\{u \in H_{\pi}^{0}, \pi\left(Y^{\beta}\right) u \in H_{\pi}^{0}\right.$ for $\left.|\beta| \leq 2\right\} . H_{\pi}^{0}$ is the space of the representation and we put the norm:

$$
u \rightarrow\left(\sum_{\mid \beta ! \leq 2} \| \pi\left(Y^{\beta}\right) u_{\cdot \mid}^{!^{2}}\right)^{1 / 2} \text { on } H_{\pi}^{2} \text { 。 }
$$

Moreover $\pi(P)$ has compact resolvent (cf. chapter II of [HE-NO $]_{3}$ ). The $\$ 1.6$ of the same chapter in this book "How to recognize an induced representation" gives a useful criterion to recognize that:

$$
H_{\alpha}=\pi_{l_{\alpha}}\left(\sum_{i=1}^{3} Y_{i}^{2}\right) \quad \text { for some } l_{\alpha} \in \mathbb{S} *
$$

where \&5 is a stratified Lie algebra with 3 generators of rank 3

Communicated by H. Araki, February 2, 1987.

* Centre de Mathématiques de l'Ecole Polytechnique, Plateau de Palaiseau-91128 Palaiseau Cedex, France.

Current address: Departement de Mathématiques et D'Informatique, Université de Nantes, Chemin de la Houssinière, 44072 Nantes Cedex 03, France. 
satisfying to

$$
\begin{gathered}
\operatorname{dim} \mathbb{S}_{1}=3, \quad \mathbb{S}_{2}=\boldsymbol{R}\left[Y_{1}, Y_{3}\right]+\boldsymbol{R}\left[Y_{2}, Y_{3}\right] \\
{\left[Y_{1}, Y_{2}\right]=0, \quad \operatorname{dim} \mathscr{S}_{3}=1}
\end{gathered}
$$

and $\pi_{l_{\alpha}}$ is the irreducible representation attached by the Kirillov map to $l_{\alpha}=(\underbrace{0,0, \alpha}_{\mathbb{S}_{1}} ; \underbrace{0,0}_{\mathbb{S}_{2}} ; \underbrace{1}_{\mathbb{S}_{3}}) \in \mathbb{S}^{*} *$

Observe now that $P$ is hypoelliptic and therefore we have (because $\left.l_{\alpha \digamma_{3}}=1\right)$ the existence of a constant $C$ such that: $\forall \alpha \in \mathbb{R}$ :

$$
\sum_{|\beta| \leq 2}\left\|\pi_{l_{\alpha}}\left(Y^{\beta}\right) u\right\|^{2} \leq C\left\|\pi_{l_{\alpha}}(P) u\right\|^{2}, \quad \forall u \in H_{\pi_{l_{\alpha}}}^{2} .
$$

That means in particular:

$$
\exists C>0, \forall \alpha \in \mathbb{R},\|u\|_{H^{2}\left(R^{2}\right)}^{2}+\sum_{0 \leq k \leq 2}\left\|\left(\alpha+x_{1} x_{2}\right)^{k} u\right\|_{L^{2}\left(R^{2}\right)}^{2} \leq C\left\|H_{\alpha} u\right\|_{L^{2}\left(R^{2}\right)}^{2} \cdot
$$

Using interpolation and the inequality

$$
\|\alpha u\|_{H_{\pi_{\alpha}}^{0}} \leq C\|u\|_{H_{\pi_{l_{\alpha}}}^{4}}
$$

we can complete (2) in proving the existence of $C>0$ s. $t \forall \alpha$

$$
(1+|\alpha|)\|u\|_{L^{2}\left(R^{2}\right)}^{2}+\sum_{0 \leq k \leq 2}\left\|\left(\alpha+x_{1} x_{2}\right)^{k} u\right\|_{L^{2}\left(R^{2}\right)}^{2} \leq C\left\|H_{\alpha} u\right\|_{L^{2}\left(R^{2}\right)}^{2} \cdot
$$

A consequence of (4) and of the trivial fact that $H_{\alpha}$ is positive is that the first eigenvalue of $H_{\alpha}$ satisfies the minoration:

$$
\lambda_{1}(\alpha) \geq \frac{1}{\sqrt{c}}[1+|\alpha|]^{1 / 2}
$$

In particular, $H_{\alpha}$ is invertible for all $\alpha$ and his spectrum is contained in $\left[\frac{1}{\sqrt{c}}[|\alpha|+1]^{1 / 2},+\infty\right]$.

Continuity of $\lambda_{n}(\alpha)$ The continuity follows from theorem 3.16 chapter IV p. 212 and remarks in $\S 3.5$ p. 213-214 in the book of Kato [KA]. It is sufficient to observe that the $H_{\alpha}$ are uniformly semi-bounded and that for each $\alpha_{0} \in \boldsymbol{R}: H_{\alpha} \rightarrow H_{\alpha_{0}}$ as $\alpha \rightarrow \alpha_{0}$ in the generalized sense i. e.

$$
\left\|H_{\alpha}^{-1}-H_{\alpha_{0}}^{-1}\right\|_{\mathscr{L}\left(L^{2}, L^{2}\right)} \underset{\alpha \rightarrow \alpha_{0}}{\longrightarrow} 0 .
$$

For $u \in \mathfrak{Z}$, we have:

$$
\left(H_{\alpha}^{-1}-H_{\alpha_{0}}^{-1}\right) u=-H_{\alpha}^{-1}\left(H_{\alpha}-H_{\alpha_{0}}\right) H_{\alpha_{0}}^{-1} u .
$$


Note that $H_{\alpha}^{-1} \in \mathfrak{L}(\mathfrak{g}, \mathfrak{Z})$; this is proved in the rank 3 case in $[\mathrm{HE}-$ $\mathrm{NO}]_{1}$, in the rank $r$ case in $[\mathrm{HE}-\mathrm{NO}]_{2}$ or in Melin [ME]). Observe now that $H_{\alpha}-H_{\alpha_{0}}=\left(\alpha-\alpha_{0}\right)^{2}+2\left(\alpha-\alpha_{0}\right)\left(\alpha_{0}+x_{1} x_{2}\right)$ and from the relations

$$
\left\{\begin{array}{l}
\left(H_{\alpha}-H_{\alpha_{0}}\right) H_{\alpha_{0}}^{-1}=2\left(\alpha-\alpha_{0}\right)\left[\left(\alpha_{0}+x_{1} x_{2}\right) H_{\alpha_{0}}^{-1}\right]+\left(\alpha-\alpha_{0}\right)^{2} H_{\alpha_{0}}^{-1} \\
\left\|\left(H_{\alpha}-H_{\alpha_{0}}\right) H_{\alpha_{0}}^{-1}\right\|_{\mathfrak{g}\left(L^{2}, L^{2}\right)} \leq \tilde{G}\left|\alpha-\alpha_{0}\right| \quad(u \operatorname{sing}(4)) \text { for }\left|\alpha-\alpha_{0}\right|<1 / \tilde{C} \\
\left\|H_{\alpha}^{-1}\right\| \leq \tilde{C}
\end{array}\right.
$$

we get

$$
|| H_{\alpha}^{-1}-H_{\alpha_{0}}^{-1} \| \leq C\left|\alpha-\alpha_{0}\right|
$$

Let us prove now that $H_{\alpha}$ is an analytic family in the sense of Kato. We have to look to the limit of

$$
\frac{H_{\alpha}^{-1}-H_{\alpha_{0}}^{-1}}{\alpha-\alpha_{0}}
$$

for $\alpha \rightarrow \alpha_{0}$ ( $\alpha$ and $\alpha_{0} \in C$ ) and to prove that this limit is: $-2 H_{\alpha_{0}}^{-1}$ $\left(\alpha_{0}+x_{1} x_{2}\right) H_{\alpha_{0}}^{-1}$. This is easy to verify if we observe that:

$$
\frac{\left(H_{\alpha}-H_{\alpha_{0}}\right) H_{\alpha_{0}}^{-1}}{\left(\alpha-\alpha_{0}\right)}-2\left(\alpha_{0}+x_{1} x_{2}\right) H_{\alpha_{0}}^{-1}=3\left(\alpha-\alpha_{0}\right) H_{\alpha_{0}}^{-1}
$$

(Here we have used that the maximal estimates (2) and (4) are true for $\alpha \in \mathbb{C}$ with $|\operatorname{Im} \alpha|<\varepsilon$ for $\varepsilon>0$ small enough).

As a corollary we get by the Kato-Rellich's theorem (Th XII 8) in [RE-SI]) and the Rellich's theorem (Th XII. 3 in [RE-SI]) that:

(7) The eigenvalues are analytic in $\alpha$ (that means that is the nhd of some $\alpha_{0}$ the eigenvalues of $H_{\alpha}$ which are near $\lambda_{n}\left(\alpha_{0}\right)$ cas be described by analytic functions of $\alpha$.

This answers to a question of $\mathrm{P}$. Jorgensen [J]. In particular:

(8) The first eigenvalue (which is simple) $\lambda_{1}(\alpha)$ is analytic.

Asymptotic for $|\alpha| \rightarrow \infty$. In a personnal letter [J] P. Jorgensen asks if the $\lambda_{n}(\alpha)$ are simple, monotone. We just write the type of results it is reasonable to prove and give the principal steps of "a proof" (we have not verified all the details).

Conjecture. For $\alpha \rightarrow+\infty$, we have 
(9) (i) $\quad \lambda_{2 k+1}(\alpha) \equiv \lambda_{2 k+2}(\alpha) \bmod \left(0\left(|\alpha|^{-\infty}\right) \quad \forall k \in \mathbb{N}\right.$

(10) (ii) $\quad \lambda_{2 k+2}(\alpha) \sim E_{2} \alpha^{1 / 2}+E_{3}(2 k+1) \alpha^{-1 / 4}+\sum_{j \geq 4} E_{j}^{k} \alpha^{2-3 j / 4} \quad \forall k \in N$.

$\begin{array}{ll} & \forall k, \forall \varepsilon>0, \exists C_{\varepsilon}^{k}>0 \\ \text { (11) (iii) } \quad\left|\lambda_{2 k+2}(\alpha)-\lambda_{2 k+1}(\alpha)\right| \geq C_{k}^{\varepsilon} e^{-\left(S_{0}+\varepsilon\right)|\alpha|^{3 / 2}}\end{array}$

with $S_{0}=4 \sqrt{2} / 3$.

Remark 1. Using the symmetry $\left(x_{1}, x_{2}\right) \rightarrow\left(-x_{1}, x_{2}\right)$, we see that:

$$
\lambda_{n}(\alpha)=\lambda_{n}(-\alpha) \quad \forall n \in \boldsymbol{N}^{*}, \forall \alpha \in \boldsymbol{R}
$$

so we hope similar results (9), (10), (11) for $\alpha \rightarrow-\infty$. \#

\section{"Proof of the conjecture"}

We make the scaling: $x_{1}=\sqrt{\alpha} y_{1}, x_{2}=-\sqrt{\alpha} y_{2}$ and we get the unitary equivalent operator:

$$
\begin{gathered}
\alpha^{2} \tilde{H}_{\alpha}\left(y, D_{y}\right) \quad \text { with } \\
\tilde{H}_{\alpha}\left(y, D_{y}\right)=\frac{1}{\alpha^{3}}\left(D_{y_{1}}^{2}+D_{y_{2}}^{2}\right)+\left(1-y_{1} y_{2}\right)^{2} .
\end{gathered}
$$

We recognize the Schrödinger operator:

$$
\tilde{H}_{\alpha}=P(h)=-h^{2} \Delta+V
$$

with $h=\alpha^{-3 / 2}$ and $V=\left(1-y_{1} y_{2}\right)^{2}$

and we are interested in studying the asymptotic behavior as $h \rightarrow 0$ of the first eigenvalues of $P(h)$.

We then follow the strategy of $[\mathrm{HE}-\mathrm{SJ}]_{1,2}$. We see that

$$
V^{-1}(0)=\left\{y_{1} y_{2}=1\right\}=U_{1} \cup U_{2}
$$

where

$$
\begin{aligned}
& U_{1}=\left\{\left(y_{1} y_{2}=1\right\} \cap y_{1}<0\right\} \\
& \left.U_{2}=\left\{\left(y_{1} y_{2}=1\right\}\right) \cap y_{1}>0\right\}
\end{aligned}
$$

$U_{1}$ and $U_{2}$ are disjoint wells and the symmetry $\left(x_{1}, x_{2}\right) \rightarrow\left(-x_{1},-x_{2}\right)$ sends $U_{1}$ on $U_{2}$ and $U_{2}$ and $U_{1}$. This explains why we have (i), and in fact we can hope that the eigenvalues $\mu_{k}(h)$ verify

$$
\left|\mu_{2 k+2}(h)-\mu_{2 k+1}(h)\right| \leq C_{k}^{\varepsilon} e^{-S_{0} / h} e^{\varepsilon / h}
$$

where $S_{0}$ is the Agmon distance attached to the metric $V d y^{2}$ between the two wells $U_{1}$ and $U_{2}$. Between $U_{1}$ and $U_{2}$ there is a unique minimal geodesic which is the straight line between $(-1,-1)$ and $(+1,+1)$ and we get: 


$$
S_{0}=\sqrt{2} \int_{-1}^{+1}\left(1-y_{1}^{2}\right) d y_{1}=4 \sqrt{2} / 3
$$

The link between $\mu_{k}(h)$ and $\lambda_{k}(\alpha)$ is given according to (13)-(15) by

$$
\lambda_{k}(\alpha)=\alpha^{2} \mu_{k}\left(\alpha^{-3 / 2}\right) \quad \text { for } \alpha>0 .
$$

According to $[\mathrm{HE}-\mathrm{S} J]_{2}$ and to be "almost" in the case treated in this paper (in $[\mathrm{HE}-\mathrm{SJ}]_{2}$ the $U_{i}$ are assumed to be compact but I think that $[\mathrm{HE}-\mathrm{SJ}]_{2}$ can be applied in the case treated here), we have a problem with two miniwells inside two wells.

Let us look to the $V_{m}^{\prime \prime}$ at a point $m$ of $U_{2}$; the eigenvalues are 0 and $2\left(y_{1}^{2}+y_{2}^{2}\right)$ where $m=\left(y_{1}, y_{2}\right)$. The non zero eigenvalue admits a minimum at $m=(1,1)$ in $U_{2}$, and it is proved in $[\mathrm{HE}-\mathrm{SJ}]_{2}$ that the eigenfunctions are localized in the miniwells $(-1,-1)$ and $(+1,+1)$.

To get (10), we have to construct $\mathrm{W}-\mathrm{K}-\mathrm{B}$ approximate eigenfunctions of the type $a(x, h) e^{-\varphi / h-\psi / \sqrt{h}}$ (see $[\mathrm{HE}-\mathrm{S} J]_{2}$ ) near $(1,1)$ (for example). As in $[\mathrm{HE}-\mathrm{SJ}]_{2}$, we get the expansion

$$
\mu_{2 k}(h) \sim E_{2} h+E_{3} h^{3 / 2}+\sum_{j \geq 4} E_{\jmath}^{k} h^{j / 2}
$$

with $E_{2}=\min \underset{\left(y_{1}, y_{2}\right) \in U_{2}}{\sqrt{y_{1}^{2}+y_{2}^{2}}}, E_{3}>0$ (In $[\mathrm{HE}-\mathrm{S} J]_{2}$, the proof is given for the first eigenvalue). The estimate (10) corresponds to

$$
\left|\mu_{2 k+2}(h)-\mu_{2 k+1}(h)\right| \geq \tilde{C}_{k}^{\varepsilon} e^{-\left(S_{0}+\varepsilon\right) / h}
$$

and corresponds to a minoration of the splitting between the eigenvalues due to the tunneling effect.

(21) is relatively clear in the case $k=0$. The case $k>0$ is probably true also adapting technics of A. Martinez [MA].

Remark 2. If the conjecture is true, for each $N_{0}, \exists C_{0}$ s.t all the eigenvalues $\lambda_{k}(\alpha)$ are simple for $k \leq N_{0}$ and $|\alpha|>C_{0}$.

If you observe the analyticity in $\alpha$, for $k \in \mathbb{N}$ fixed then $\lambda_{k}(\alpha)$ is simple and analytic outside a finite number of points. \#

Remark 3. Let us give now a slightly different approach to localize the $\lambda_{k}(\alpha)$ as $\alpha \rightarrow \pm \infty$. The results are weaker but the proof is relatively general and uses group theoretical methods. Let us consider the operator $\frac{1}{\sqrt{\alpha}} H_{\alpha}$ for $\alpha>0$. We have $\frac{1}{\sqrt{\alpha}} \cdot H_{\alpha}=\pi_{l_{\alpha}}\left(\sum_{i=1}^{3} Y_{2}^{2}\right)$ with 
$\tilde{l}_{\alpha}=\left(0,0, \alpha^{3 / 4}, 0,0, \alpha^{-1 / 2}\right)$. Associated to $\tilde{l}_{\alpha}$, let us consider the orbit of $\tilde{l}_{\alpha}$ :

$$
\begin{aligned}
& G . \tilde{l}_{\alpha}=\left\{l \in \mathbb{S}^{*} \text { s. } \mathrm{t} \exists\left(\eta_{1}, \eta_{2}, y_{1}, y_{2}\right) \in \mathbb{R}^{q}\right. \text { s. t } \\
&\left(\eta_{1}, \eta_{2}, \alpha^{3 / 4}\left(1+y_{1} y_{2}\right), y_{1}, y_{2}, \alpha^{-3 / 4}\right) .
\end{aligned}
$$

Let us introduced the set

$$
\mathfrak{L}\left(\tilde{l}_{\alpha}\right)=\left\{l \in \mathbb{S} * \text { s. } \mathrm{t} \exists \alpha_{n} \text { with } \alpha_{n} \rightarrow \infty \text { and } l^{(n)} \in\left(G l_{\alpha_{n}}\right) \text { s.t } l^{(n)} \rightarrow l\right\} .
$$

Then using the technics of Helffer-Nourrigat $[\mathrm{HE}-\mathrm{NO}]_{3}$ (see Theorem 4.9 in $\left.[\mathrm{HE}]_{2}\right)$ it is possible to prove that

$$
\lim _{|\alpha| \rightarrow \infty} \frac{\lambda_{k}(\alpha)}{\sqrt{\alpha}} \in \overline{\bigcup_{l \in \mathbb{R}\left(l_{\alpha}\right)} S p \pi_{l}\left(\sum Y_{i}^{2}\right)} .
$$

This type of argument appears in similar contexts in $[\mathrm{HE}]_{1}$ (after Helffer-Métivier-Nourrigat) and in [HE-MO].

Let us just describe $\mathfrak{L}\left(\tilde{l}_{\alpha}\right)$ and the family of the $S p\left(\pi_{l}\left(\sum Y_{l}^{2}\right)\right)$

$$
\mathfrak{L}\left(\tilde{l}_{\alpha}\right)=\left\{l \in \mathbb{S S}^{*} \text { s.t } \exists\left(\eta_{1}, \eta_{2}, \eta_{3}, y_{1}, y_{2}\right) \in \mathbb{R}^{4}\right.
$$

with $y_{1} y_{2}=-1$ and

$$
\left.l=\left(\eta_{1}, \eta_{2}, \eta_{3}, y_{1}, y_{2}, 0\right)\right\}
$$

Then it is not to difficult to verify that $S p\left(\Pi_{l}\left(\sum_{2=1}^{n} Y_{2}^{2}\right)\right)=\left[\sqrt{y_{1}^{2}+y_{2}^{2}},+\right.$ $\infty]$.

As a corollary we get that for $|\alpha|>\alpha_{0}$ we have: $\lambda_{1}(\alpha) \geq \sqrt{2}|\alpha|^{1 / 2}$ which is coherent with (19) and (20).

I want to thank P. Jorgensen and A. Martinez for valuable discussions.

\section{References}

[HE] B. Helffer,

[1] On the hypoellipticity of operators of the type

$$
\sum_{j=1}^{n} Y_{j}^{2}+\frac{1}{2} \sum_{j, k} C_{j k}\left[Y_{j}, Y_{k}\right]
$$

Séminaire de I'Université de Nantes exposé $\mathrm{n}^{\circ} 1,1981-82$.

[2] Partial differential equations on nilpotent groups, Lecture Notes in Mathematics, $\mathrm{n}^{\circ} 1077,210-254$.

Lie group representations III.

[HE-MO] B. Helffer-A. Mohamed, Séminaire de I'Université de Nautes 86-87.

[HE-NO] B. Helffer-J. Nourrigat,

[1] Hypoellipticité pour des groupes de rang 3, Comm. in P.D.E., 3 (8) (1978), 693-743.

[2] Théorèmes d'indice pour des opérateurs différentiels à coefficients polynomiaux, 
Partial differential equations, Banach Center publications, 10 (1983).

[3] Hypoellipticité maximale pour des opérateurs polynômes de champs de vecteurs, Progress in Matbematics, 58 Birkhaüser.

[HE-SJ] B. Helffer-J. Sjöstrand,

[1] Multiple Wells in the semiclassical limit I, Comm. In P. D. E. 9 (4) (1984), 337-408.

[2] Puits multiples en mécanique semi-classique $\mathrm{V}$ (étude des minipuits), Volume in Honor of S. Mizohata 1986, See also séminaire de Nantes 1984-85 Exposé $\mathrm{n}^{\circ} 5$ and séminaire à I'Ecole Polytechnique 1985-86 Exposé n ${ }^{\circ} 10$.

[J] P. Jorgensen, Personnal communication.

[J-K] P. Jorgensen-W. Klink, Quantum Mechanics and Nilpotent Groups I. The curved magnetic field, Publ. RIMS Kyoto Univ., 21, (1985), 969-999.

[KA] T. Kato, Perturbation theory for linear operators, Springer.

[MA] A. Martinez, Estimation de l'effet tunnel pour le double puits, Publications Mathématiques d'Orsay, 85T17 (to appear in Journal de Mathématiques pures et appliquées).

[MEL] A. Melin, Parametrix constructions, Ann. Glob. Analysis and Geometry $\mathbb{1}$ (1983), 79-130.

[RE-SI] M. Reed-B. Simon, Methods of Modern Mathematical Physics Tome IV, Analysis of operators, Academic Press, Inc., 1978. 
